\title{
Regularizing future cosmological singularities with varying speed of light
}

\author{
F. Shojai ${ }^{1,2, a}$, A. Shojai ${ }^{1,2}$, M. Sanati ${ }^{1}$ \\ ${ }^{1}$ Department of Physics, University of Tehran, Tehran, Iran \\ ${ }^{2}$ Foundations of Physics Group, School of Physics, Institute for Research in Fundamental Sciences (IPM), Tehran, Iran
}

Received: 10 July 2015 / Accepted: 13 November 2015 / Published online: 30 November 2015

(C) The Author(s) 2015. This article is published with open access at Springerlink.com

\begin{abstract}
Cosmological models may result in future singularities. Using scalar-tensor gravity as the dynamical theory for describing a varying speed of light, we show that it is possible to regularize those singularities.
\end{abstract}

\section{Introduction}

The current observational data, interpreted in the framework of FLRW cosmology, indicates that the dominant content of the universe is in the form of dark energy whose equation of state parameter, $w$ is close to -1 . It is quite possible that dark energy scenarios drive the universe toward some type of singularity. Fore example, in a simple case with a time independent phantom equation of state parameter $(w<-1)$, the universe evolves toward a big-rip singularity [1]. This kind of singularity is an example of other new exotic singularities that may happen in the future of the universe. These singularities usually violate some of the energy conditions. They can be classified according to their properties. This classification of the future cosmological singularities is summarized in the next section.

An important question is if is it possible to find a mechanism to change the nature of these singularities or remove them. This question was recently investigated in the framework of varying physical constants theories [2,3]. Some examples are $G$-variable theory [4], $e$-variable theory [5], $c$-variable theory [6-8] and $G$-c-variable theory $[9,10]$. The last two play an important role in the special and general relativity. A naive introduction of a variable speed of light breaks Lorentz invariance. There would be a preferred frame, in which Einstein's equations are valid in which the time dependent speed of light is inserted as input. But it can be shown [11] that by introducing a new time-like coordinate, one can retrieve local Lorentz invariance and general covari-

ae-mail: fshojai@ut.ac.ir ance. Varying speed of light theories can be motivated by solving the problems of standard cosmological model. It is shown that a larger speed of light in the early universe can solve both horizon and flatness problems [6-8].

In this paper we investigate how a varying speed of light theory can regularize some of the future finite-time cosmological singularities [2]. We use a general scalar tensor action in which the dynamical speed of light is coupled to gravity non-minimally. Then we shall find the necessary coupling and the potential function to regularize some of the future singularities.

\section{Future singularities}

As stated before, one of the features of a dark energy dominated universe is the possibility of appearance of future exotic singularities, leading to the violation of energy conditions. For later convenience we review the classification of such singularities [12-14]:

- Type I (big-rip singularity): For $t \rightarrow t_{\mathrm{s}}, a \rightarrow \infty, \rho \rightarrow \infty$, and $|p| \rightarrow \infty$.

This singularity happens in a phantom dark energy model with an equation of state parameter $w<-1[15]$ and all the energy conditions are violated.

- Type II (sudden future singularity): For $t \rightarrow t_{\mathrm{s}}, a \rightarrow a_{\mathrm{s}}$, $\rho \rightarrow \rho_{\mathrm{s}}$, and $|p| \rightarrow \infty$.

This singularity was first investigated by Barrow [16]. He constructed a class of models which leads to sudden future singularity for which the weak and strong energy conditions hold but the dominant energy condition is violated. This is a pressure singularity that keeps the density, the scale factor, and the Hubble parameter finite.

- Type III (finite scale factor or big freeze singularity): For $t \rightarrow t_{\mathrm{s}}, a \rightarrow a_{\mathrm{s}}, \rho \rightarrow \infty$, and $|p| \rightarrow \infty$. 
This singularity has been discovered in the model of [17] and then found in [18] for phantom models with generalized Chaplygin gas.

- Type IV (big-brake or big-separation singularity): For $t \rightarrow$ $t_{\mathrm{s}}, a \rightarrow a_{\mathrm{s}}, \rho \rightarrow 0,|p| \rightarrow 0$ but derivatives of the Hubble parameter may diverge.

Such a singularity can be found in the tachyonic cosmological model [19].

- Generalized sudden future singularities: For $t \rightarrow t_{\mathrm{S}} a \rightarrow$ $a_{\mathrm{s}}, \rho \rightarrow \rho_{\mathrm{s}},|p| \rightarrow p_{\mathrm{s}}$, and the derivatives of the pressure may diverge [20]. For this singularity, all the energy conditions are satisfied.

- $w$-singularity: For $t \rightarrow t_{\mathrm{s}}, a \rightarrow a_{\mathrm{s}}, \rho \rightarrow 0,|p| \rightarrow 0$, the equation of state parameter diverges while the derivatives of the Hubble parameter do not. The energy conditions do not seem to violate at this singularity [21].

Moreover, in [22] a number of different finite-time singularities are studied using generalized power series expansion of the scale factor. The energy conditions are analyzed in the vicinity of these events. For a $w$ singularity the scale factor admits a Taylor series in which the linear and quadratic terms are absent [23].

From a different point of view, the authors of [24] discuss the behavior of geodesics in the presence of a variety of cosmological singularities. In this way they are able to classify singularities into two groups, weak and strong. In the former case the space-time is geodesically complete and in the latter is incomplete.

\section{Regularization of future singularities in varying $c$ models}

In order to investigate the possibility of regularization of future singularities within varying speed of light theories, we use the scale factor recently proposed by Dabrowski and Marosek [2]. It admits a variety of singularities by adjusting the range of some parameters. It is given by

$a(t)=a_{\mathrm{s}}\left(\frac{t}{t_{\mathrm{S}}}\right)^{m} \exp \left(1-\frac{t}{t_{\mathrm{s}}}\right)^{n}$

where $m$ and $n$ are arbitrary constants. $t_{\mathrm{s}}$ and $a_{\mathrm{s}}$ are the finite cosmic time and the scale factor at which a particular singularity may occur depending on the values of $m$ and $n$. By this form of the scale factor, the universe has started with a big-bang singularity at $t=0$ and eventually can reach another singularity at the future. According to Dabrowski and Marosek [2], the possible future singularities of the above cosmological model can be classified as shown in Table 1.

It is quiet acceptable to believe that time variation of physical constants, especially the speed of light, may regularize some of the cosmological singularities. There are two ways
Table 1 Future singularities of the cosmological model given by Eq. (1)

\begin{tabular}{lll}
\hline$n$ & $m$ & Singularity \\
\hline$(1,2)$ & Arbitrary & Sudden future (type II) \\
Arbitrary & $(-\infty, 0)$ & Big rip (type I) \\
$(0,1)$ & Arbitrary & Finite scale factor (type III) \\
$n>2$ & 0 & $w$ \\
\hline
\end{tabular}

of making a physical constant time dependent. The naive way is to feed time dependence of any physical constant as an input function in the equations of motion of the theory. Thus the basic postulate of this method is that the equations of motion are valid with variable constants but this can only be true in one frame. This method was proposed in [6-8] as a solution to some of the problems of the standard cosmological model. Recently, it was shown that this way of forcing the speed of light or the gravitational constant to vary can regularize some of the future singularities [2,3,25]. In fact, in these papers appropriate time dependent constants are suggested such that some of the singularities, types II and III, are regularized.

To have a physically meaningful regularization, it should be covariant. Therefore the regularization should be done in dynamically variable constant theories. Here we are interested to regularize the type II, III and $w$ singularities by varying speed of light.

As Ellis and Uzan [26] pointed out, to change a constant into a dynamical variable, one needs to start from a new Lagrangian in which the constant is replaced by a dynamical field with the corresponding dynamical terms. One of the simplest actions suitable for a dynamical speed of light is that of the scalar-tensor model. In the Jordan picture it is $[27,28]$ :

$$
\begin{aligned}
S= & \frac{1}{16 \pi G} \int \mathrm{d}^{4} x \sqrt{-g} \\
& \times\left(F(\Psi) R-2 U(\Psi)-g^{\mu \nu} \partial_{\mu} \Psi \partial_{\nu} \Psi\right) \\
& +S_{\mathrm{m}}\left[\phi_{i}, g_{\mu \nu}\right]
\end{aligned}
$$

in which we have to assume $F=\left(c / c_{0}\right)^{4}$ and $\mathrm{d}^{4} x=\mathrm{d} t \mathrm{~d}^{3} x$ to have the correct limit of constant speed of light. $U(\Psi)$ is the potential energy of the scalar field $\Psi, c_{0}$ is the constant velocity of light and hereafter we shall put $8 \pi G=c_{0}^{4}=1$. $S_{\mathrm{m}}$ is the action of the matter fields, $\phi_{i}$, and does not involve the $\Psi$ field, that is to say, we have assumed that the matter is minimally coupled to gravity.

Note that there are three ways of interpreting the coupling function $F(\Psi)$. First, as stated above, looking at this as a $G$-constant, $c$-variable model. Second, it can represent a $G$ variable, $c$-constant model. The regularization method and the results that will be obtained later do not differ for these 
two cases. The only difference is that the $c$-variable model breaks the local Lorentz invariance but $G$-variable model saves it. The third case is to consider this as a $G$-c-variable model like the model of Balcerzak $[9,10]$. The difference is that in the present model, the action (2) has an arbitrary coupling function $F(\Psi)$ and potential $U(\Psi)$, and thus it is a generalization of Brans-Dicke theory. But in the action of Balcerzak, the coupling function is not arbitrary (it is a fixed function of $G$ and $c$ coupled to both potential and kinetic terms). Moreover, the form of the potential function is fixed.

Variation of the action (2) with respect to the metric and the scalar field gives

$$
\begin{aligned}
& F(\Psi)\left(R_{\mu \nu}-\frac{1}{2} g_{\mu \nu} R\right) \\
& =T_{\mu \nu}^{\text {matter }}+\nabla_{\mu} \Psi \nabla_{\nu} \Psi-\frac{1}{2} g_{\mu \nu}\left(\nabla_{\alpha} \Psi\right)^{2} \\
& \quad+\nabla_{\mu} \nabla_{\nu} F(\Psi)-g_{\mu \nu} \nabla_{\mu} \nabla^{\mu} F(\Psi)-g_{\mu \nu} U(\Psi), \\
& \nabla_{\alpha} \nabla^{\alpha} \Psi=-\frac{1}{2} \frac{\mathrm{d} F}{\mathrm{~d} \Psi} R+\frac{\mathrm{d} U}{\mathrm{~d} \Psi},
\end{aligned}
$$

and variation with respect to the matter fields gives the matter equations of motion.

Applying the field equations (3)-(4) to the FLRW universe, we get

$$
\begin{aligned}
& 3 F\left(H^{2}+\frac{k}{a^{2}}\right)=\rho+\frac{1}{2} \dot{\Psi}^{2}-3 H \dot{F}+U, \\
& -2 F\left(\dot{H}-\frac{k}{a^{2}}\right)=(\rho+p)+\dot{\Psi}^{2}+\ddot{F}-H \dot{F}, \\
& (\ddot{\Psi}+3 H \dot{\Psi})=3 \frac{\mathrm{d} F}{\mathrm{~d} \Psi}\left(\dot{H}+2 H^{2}+\frac{k}{a^{2}}\right)-\frac{\mathrm{d} U}{\mathrm{~d} \Psi} .
\end{aligned}
$$

The first two equations are varying speed of light-FLRW equations and the other is the equation of motion of $\Psi$ field. $H, \rho$, and $p$ are the Hubble parameter, energy, and pressure densities of the matter field and a dot denotes a derivative with respect to the time-like coordinate $t$. From (1), the Hubble parameter and its derivative are

$$
\begin{aligned}
& H(t)=\frac{m}{t}-\frac{n}{t_{\mathrm{s}}}\left(1-\frac{t}{t_{\mathrm{s}}}\right)^{n-1}, \\
& \dot{H}(t)=-\frac{m}{t^{2}}+\frac{n(n-1)}{t_{\mathrm{s}}^{2}}\left(1-\frac{t}{t_{\mathrm{s}}}\right)^{n-2} .
\end{aligned}
$$

In order to regularize the singularities, let us assume that the time dependence of the coupling function $F$ and the field $\Psi$ are given by

$$
\begin{aligned}
& F=F_{0}\left(1-\frac{t}{t_{\mathrm{s}}}\right)^{\beta} \\
& \Psi=\Psi_{\mathrm{s}}\left(\frac{t}{t_{\mathrm{s}}}\right)^{\alpha}+\Psi_{0}\left(1-\frac{t}{t_{\mathrm{s}}}\right)^{\gamma}
\end{aligned}
$$

where $F_{0}, \Psi_{\mathrm{s}}, \Psi_{0}, \beta, \alpha$, and $\gamma$ are arbitrary constants. By substituting (10) and (11) into (5), and (6), we find that

$$
\begin{aligned}
& \rho(t)=3 F_{0}\left[\frac{m^{2}}{t^{2}}\left(1-\frac{t}{t_{\mathrm{S}}}\right)^{\beta}+\frac{n^{2}}{t_{\mathrm{S}}^{2}}\left(1-\frac{t}{t_{\mathrm{S}}}\right)^{\beta+2(n-1)}\right. \\
& \left.-\frac{2 m n}{t t_{\mathrm{s}}}\left(1-\frac{t}{t_{\mathrm{s}}}\right)^{\beta+n-1}\right] \\
& -\frac{\alpha^{2} \Psi_{\mathrm{s}}^{2}}{2 t_{\mathrm{s}}^{2}}\left(\frac{t}{t_{\mathrm{s}}}\right)^{2(\alpha-1)}-\frac{\Psi_{0}^{2} \gamma^{2}}{2 t_{\mathrm{s}}^{2}}\left(1-\frac{t}{t_{\mathrm{s}}}\right)^{2(\gamma-1)} \\
& +\frac{\Psi_{0} \Psi_{\mathrm{s}} \alpha \gamma}{t_{\mathrm{s}}^{2}}\left(\frac{t}{t_{\mathrm{s}}}\right)^{\alpha-1}\left(1-\frac{t}{t_{\mathrm{s}}}\right)^{\gamma-1} \\
& -\frac{3 F_{0} \beta}{t_{\mathrm{s}}}\left[\frac{m}{t}\left(1-\frac{t}{t_{\mathrm{s}}}\right)^{\beta-1}-\frac{n}{t_{\mathrm{S}}}\left(1-\frac{t}{t_{\mathrm{s}}}\right)^{\beta+n-2}\right] \\
& -U(t) \text {, } \\
& P(t)=F_{0}\left[\frac{m(2-3 m)}{t^{2}}\left(1-\frac{t}{t_{\mathrm{s}}}\right)^{\beta}\right. \\
& -\frac{2 n(n+\beta-1)}{t_{\mathrm{S}}^{2}}\left(1-\frac{t}{t_{\mathrm{S}}}\right)^{\beta+n-2} \\
& -\frac{3 n^{2}}{t_{\mathrm{S}}^{2}}\left(1-\frac{t}{t_{\mathrm{S}}}\right)^{\beta+2(n-1)} \\
& +\frac{6 m n}{t_{\mathrm{s}}^{2}}\left(1-\frac{t}{t_{\mathrm{s}}}\right)^{\beta+n-1}+\frac{2 \beta m}{t t_{\mathrm{s}}}\left(1-\frac{t}{t_{\mathrm{s}}}\right)^{\beta-1} \\
& \left.-\frac{\beta(\beta-1)}{t_{\mathrm{S}}^{2}}\left(1-\frac{t}{t_{\mathrm{S}}}\right)^{\beta-2}\right] \\
& -\frac{1}{2 t_{\mathrm{S}}^{2}}\left[\alpha^{2} \Psi_{\mathrm{s}}^{2}\left(\frac{t}{t_{\mathrm{s}}}\right)^{2(\alpha-1)}+\Psi_{0}^{2} \gamma^{2}\left(1-\frac{t}{t_{\mathrm{S}}}\right)^{2(\gamma-1)}\right. \\
& \left.-2 \Psi_{0} \Psi_{\mathrm{s}} \alpha \gamma\left(\frac{t}{t_{\mathrm{s}}}\right)^{\alpha-1}\left(1-\frac{t}{t_{\mathrm{s}}}\right)^{\gamma-1}\right]+U(t) .
\end{aligned}
$$

Since for a sudden future singularity $1<n<2$, in order to get finite values of the density and pressure it is required that $\beta \geq 2$ and $\gamma \geq 1$ and the potential term contains positive powers of $\left(1-\frac{t}{t_{\mathrm{s}}}\right)$. Keeping this in mind, let us to find the appropriate form of potential from (7) to regularize sudden future singularity. Multiplying (7) by $\dot{\Psi}$, the time derivative of the potential is easily found as follows:

$$
\begin{aligned}
\dot{U}(t)= & \frac{3 F_{0} \beta m(1-2 m)}{t^{2} t_{\mathrm{s}}}\left(1-\frac{t}{t_{\mathrm{s}}}\right)^{\beta-1} \\
& +\frac{\Psi_{0} \Psi_{\mathrm{s}} \alpha \gamma}{t_{\mathrm{s}}^{2}}\left[\frac{\alpha-1}{t_{\mathrm{s}}}+\frac{6 m t_{\mathrm{s}}}{t^{2}}\right]\left(\frac{t}{t_{\mathrm{s}}}\right)^{\alpha-2}\left(1-\frac{t}{t_{\mathrm{s}}}\right)^{\gamma-1} \\
& -\frac{3 F_{0} \beta}{t_{\mathrm{s}}}\left[\frac{n(n-1)}{t_{\mathrm{s}}^{2}}\left(1-\frac{t}{t_{\mathrm{s}}}\right)^{n+\beta-3}\right.
\end{aligned}
$$




$$
\begin{aligned}
& +\frac{2 n^{2}}{t_{\mathrm{s}}^{2}}\left(1-\frac{t}{t_{\mathrm{s}}}\right)^{2 n+\beta-3} \\
& \left.-\frac{4 m n}{t}\left(1-\frac{t}{t_{\mathrm{s}}}\right)^{\beta+n-2}\right] \\
& -\frac{1}{t_{\mathrm{s}}^{3}}\left[\Psi_{\mathrm{s}}^{2} \alpha^{2}(\alpha-1)\left(\frac{t}{t_{\mathrm{s}}}\right)^{2 \alpha-3}\right. \\
& +\Psi_{\mathrm{s}} \Psi_{0} \alpha \gamma(\gamma-1)\left(\frac{t}{t_{\mathrm{s}}}\right)^{\alpha-1}\left(1-\frac{t}{t_{\mathrm{s}}}\right)^{\gamma-2} \\
& \left.-\Psi_{0}^{2} \gamma^{2}(\gamma-1)\left(1-\frac{t}{t_{\mathrm{s}}}\right)^{2 \gamma-3}\right] \\
& -\frac{3}{t_{\mathrm{s}}^{2}}\left[\frac{\Psi_{\mathrm{s}}^{2} \alpha^{2} m}{t}\left(\frac{t}{t_{\mathrm{s}}}\right)^{2(\alpha-1)}\right. \\
& +\frac{m \Psi_{0}^{2} \gamma^{2}}{t}\left(1-\frac{t}{t_{\mathrm{s}}}\right)^{2(\gamma-1)} \\
& -\frac{n \Psi_{\mathrm{s}}^{2} \alpha^{2}}{t_{\mathrm{s}}}\left(\frac{t}{t_{\mathrm{s}}}\right)^{2(\alpha-1)}\left(1-\frac{t}{t_{\mathrm{s}}}\right)^{n-1} \\
& -\frac{n \Psi_{0}^{2} \gamma^{2}}{t_{\mathrm{s}}}\left(1-\frac{t}{t_{\mathrm{s}}}\right)^{2 \gamma+n-3} \\
& \left.+\frac{2 \Psi_{\mathrm{s}} \Psi_{0} \alpha \gamma n}{t_{\mathrm{s}}}\left(\frac{t}{t_{\mathrm{s}}}\right)^{\alpha-1}\left(1-\frac{t}{t_{\mathrm{s}}}\right)^{n+\gamma-2}\right] \text {. }
\end{aligned}
$$

At the vicinity of $t_{\mathrm{s}}$, integrating the dominant terms of the above expression gives

$$
\begin{aligned}
U(t)= & U_{0} \\
& + \begin{cases}\frac{\Psi_{\mathrm{s}}^{2} \alpha^{2}(\alpha-1+3 m)}{t_{\mathrm{s}}^{2}}\left(1-\frac{t}{t_{\mathrm{s}}}\right) & \text { if } \gamma>2, \\
\frac{\Psi_{\mathrm{s}} \Psi_{0} \alpha \gamma}{t_{\mathrm{s}}^{2}}\left(1-\frac{t}{t_{\mathrm{s}}}\right)^{\gamma-1} & \text { if } \gamma<2,\end{cases}
\end{aligned}
$$

where $U_{0}$ is an integration constant. In this limit the energy and pressure densities are

$$
\begin{aligned}
\rho(t) & =-\left(U_{0}+\frac{\Psi_{\mathrm{s}}^{2} \alpha^{2}}{2 t_{\mathrm{s}}^{2}}\right)+\frac{\Psi^{2} \alpha^{2}(1-6 m)}{2 t_{\mathrm{s}}^{2}}\left(1-\frac{t}{t_{\mathrm{s}}}\right), \\
P(t) & =\left(U_{0}-\frac{\Psi_{\mathrm{s}}^{2} \alpha^{2}}{2 t_{\mathrm{s}}^{2}}\right) \\
& + \begin{cases}\frac{-F_{0} \beta(\beta-1)}{t_{\mathrm{s}}^{2}}\left(1-\frac{t}{t_{\mathrm{s}}}\right)^{\beta-2} & \text { if } \beta<1+\gamma, \beta<3, \\
\frac{2 \Psi_{0} \Psi_{\mathrm{s}} \alpha \gamma}{t_{\mathrm{s}}^{2}}\left(1-\frac{t}{t_{\mathrm{s}}}\right)^{\gamma-1} & \text { if } \beta>1+\gamma, \gamma<2 \\
\frac{\Psi_{\mathrm{s}}^{2} \alpha^{2}(\alpha-1+3 m)}{t_{\mathrm{s}}^{2}}\left(1-\frac{t}{t_{\mathrm{s}}}\right) & \text { if } \beta>3, \gamma>2 .\end{cases}
\end{aligned}
$$

From (16) and (17) one can find that for $\beta \geq 2$ and $\gamma \geq 1$, the energy density and pressure vanish at $t_{\mathrm{s}}$. Moreover, a sudden future singularity is regularized and the equation of state parameter of matter is in this limit
$w=-\frac{U_{0}-\frac{\Psi_{\mathrm{s}}^{2} \alpha^{2}}{2 t_{\mathrm{s}}^{2}}}{U_{0}+\frac{\Psi_{\mathrm{s}}^{2} \alpha^{2}}{2 t_{\mathrm{s}}^{2}}}$

which is finite. The varying speed of light is $c_{0} F_{0}^{1 / 4}\left(1-\frac{t}{t_{\mathrm{s}}}\right)^{\beta}$, which also approaches zero in the vicinity of $t_{\mathrm{s}}$. This is in accordance to the result of [2]. Moreover, slowing and stopping of light is also predicted in loop quantum cosmology [29]. From (11), one can conclude that for $\gamma>1$, at $t \rightarrow t_{\mathrm{s}}$,

$\alpha\left(1-\frac{t}{t_{\mathrm{s}}}\right)=1-\frac{\Psi}{\Psi_{\mathrm{s}}}$.

Substituting this in (15), the $\psi$-dependence of the coupling and potential function results.

One can repeat all of the above discussion for the other singularities. We will not pursue this calculation here and only report the results. For a finite scale factor singularity, the potential, density, and pressure functions are finite if $\beta \geq 2$ and $\gamma \geq 1$. But since $0<n<1$, at the vicinity of $t_{\mathrm{s}}$,

$$
\begin{aligned}
U(t)= & U_{0} \\
& + \begin{cases}\frac{3 F_{0} \beta n(n-1)}{t_{\mathrm{s}}^{2}(n+\beta-2}\left(1-\frac{t}{t_{\mathrm{s}}}\right)^{n+\beta-2} & \text { if } n+\beta<\gamma+1, \\
\frac{\Psi_{\mathrm{s}} \Psi_{0} \alpha \gamma}{t_{\mathrm{s}}^{2}}\left(1-\frac{t}{t_{\mathrm{s}}}\right)^{\gamma-1} & \text { if } n+\beta>\gamma+1,\end{cases}
\end{aligned}
$$

$\rho(t)=-\left(U_{0}+\frac{\Psi_{\mathrm{s}}^{2} \alpha^{2}}{2 t_{\mathrm{S}}^{2}}\right)+\frac{3 F_{0} \beta n(\beta-1)}{t_{\mathrm{s}}^{2}(n+\beta-2)}\left(1-\frac{t}{t_{\mathrm{s}}}\right)^{n+\beta-2}$,

and $P(t)$ is given by (17).

Also for the $w$ singularity, $m=0$ and $n>2$. In this case the potential and density functions are given by (15) and (16) with $m=0$, respectively.

\section{Conclusion}

We have considered a general scalar-tensor theory in which the varying speed of light is a function of the scalar field. Applying this theory to the Friedmann cosmology, we have shown that one can regularize some kinds of singularities including sudden future, finite scale factor, and $w$ singularities. To do this, we have assumed a simple time dependence for the coupling function and the scalar field. We have found that sudden future, finite scale factor, and $w$ singularities can be regularized by a varying $c(t)$ which is zero at the vicinity of these singularities. It is interesting to note that using the obtained forms of density and pressure in the vicinity of regularized singularity, one can simply observe that the energy conditions are broken. This is because at $t_{\mathrm{s}}$, the quantity $\rho+P$ approaches $-\Psi_{\mathrm{s}}^{2} \alpha^{2} / t_{\mathrm{s}}^{2}$, which is negative and this breaks all the energy conditions. 
Acknowledgments This work is supported by a grant from the university of Tehran.

Open Access This article is distributed under the terms of the Creative Commons Attribution 4.0 International License (http://creativecomm ons.org/licenses/by/4.0/), which permits unrestricted use, distribution, and reproduction in any medium, provided you give appropriate credit to the original author(s) and the source, provide a link to the Creative Commons license, and indicate if changes were made. Funded by SCOAP ${ }^{3}$.

\section{References}

1. R.R. Caldwell, M. Kamionkowski, N.N. Weinberg, Phys. Rev. Lett. 91, 071301 (2003)

2. M.P. Dabrowski, K. Marosek, JCAP 02, 012 (2013)

3. M.P. Dabrowski, K. Marosek, A. Balcerzak, Proceedings of the Sesto conference on varying fundamental constants and dynamical dark energy, Sesto (2013). arXiv: 1308.5462

4. C. Brans, R. Dicke, Phys. Rev. 124, 925 (1961)

5. J.D. Bekenstein, Phys. Rev. D 25, 1527 (1982)

6. J.W. Moffat, Int. J. Mod. Phys. D 2, 351 (1993)

7. A. Albrecht, J. Magueijo, Phys. Rev. D 59, 043516 (1999)

8. J.D. Barrow, Phys. Rev. D 59, 043515 (1999)

9. A. Balcerzak, JCAP 04, 019 (2015)
10. K. Leszczynska, A. Balcerzak, M.P. Dabrowski, JCAP 02, 012 (2015)

11. J. Magueijo, Phys. Rev. D 62, 103521 (2000)

12. E.J. Copeland, M. Sami, S. Tsujikawa, Int. J. Mod. Phys. D 15, 1753 (2006)

13. S. Nojiri, S.D. Odintsov, S. Tsujikawa, Phys. Rev. D 71, 063004 (2005)

14. M.P. Dabrowski, T. Denkiewicz, AIP Conf. Proc. 1241, 561 (2010)

15. R.R. Caldwell, Phys. Lett. B 545, 23 (2002)

16. J.D. Barrow, Class. Quant. Grav. 21, L79 (2004)

17. S. Nojiri, S.D. Odintsov, Phys. Rev. D 70, 103522 (2004)

18. M. Bouhmadi-Lopez, P.F. Gonzalez-Diaz, P. Martin-Moruno, Phys. Lett. B 659, 1 (2008)

19. V. Gorini, A. Kamenshchik, U. Moschella, V. Pasquier, Phys. Rev. D 69, 123512 (2004)

20. J.D. Barrow, C.G. Tsagas, Class. Quant. Grav. 22, 1563 (2005)

21. M.P. Dabrowski, T. Denkiewicz, Phys. Rev. D 79, 063521 (2009)

22. C. Cattoen, M. Visser, Class. Quant. Grav. 22, 4913 (2005)

23. L. Fernandez-Jambrina, Phys. Rev. D 82, 124004 (2010)

24. L. Fernandez-Jambrina, R. Lazkoz, Phys. Rev. D 74, 064030 (2006)

25. M.P. Dabrowski, T. Denkiewicz, C.J.A.P. Martins, P.E. Vielzeuf, Phys. Rev. D 89, 123512 (2014)

26. G.F.R. Ellis, J.P. Uzan, Am. J. Phys. 73(3), 240 (2005)

27. G. Esposito-Farese, D. Polarski, Phys. Rev. D 63, 063504 (2001)

28. A. Riazuelo, J.P. Uzan, Phys. Rev. D 66, 023525 (2002)

29. T. Cailleteau, J. Mielczarek, A. Barrau, J. Grain, Class. Quantum Grav. 29, 095010 (2012) 